\title{
Abandono y permanencia en la educación superior: Una aplicación de la Teoría Fundamentada*
}

\section{Abandonment and permanency in higher education: An application of Founded Theory}

\author{
**PhD. Profesor adscrito al departamento de Ciencias Hu- \\ manas, Sociales e Idiomas. jesusurbina@ufps.edu.co Gru- \\ po de investigación en estudios sociales y pedagogía para la \\ Jesús Ernesto Urbina Cárdenas** \\ Gustavo Adolfo Ovalles Rodriguez*** \\ paz de la UFPS, Cúcuta. \\ *** Mg. Docente catedrático de la UFPS (www.ufps.edu. \\ co).gustavoadolfo@ufps.edu.co \\ Cómo citar: Urbina JE \& Ovalle G.A. (2016) Abandono y \\ permanencia en la educación superior: Una aplicación de la \\ teoría fundamentada.Sophia 12(1): 27-37.
}

\section{Resumen}

Siendo el abandono académico un problema de gran trascendencia para los sistemas educativos y para la sociedad en general, el presente estudio se propone la comprensión de los factores pedagógicos que inciden en el abandono y permanencia de los estudiantes del Programa de Ingeniería Industrial de la Universidad Francisco de Paula (UFPS. Se fundamenta teóricamente en el Modelo de Integración Estudiantil de Tinto. La investigación es de corte comprensivo y utiliza como apoyo la Teoría Fundamentada (Strauss \& Corbin, 2006). Los resultados muestran la motivación como el factor fundamental que impulsa la integración académica y social del estudiante y que culmina con la adaptación a la vida universitaria. Este proceso de motivación es promovido por las relaciones que establece el estudiante con su círculo de amigos, y recibe la influencia directa de las acciones pedagógicas desarrolladas por los profesores, el programa y la universidad.

Palabras Clave: Abandono escolar, motivación, pedagogía, teoría fundamentada, ciencias de la educación.

\begin{abstract}
Academic abandonment being a great problem for education systems, and for the society in general, the purpose of this study is to understand pedagogic factors which impact abandonment and permanency of students of Industrial Engineering Program at Francisco de Paula University - UFPS. Theoretically founded on the Student Integration Model Tinto. It is a comprehensive research, and uses the Founded Theory as a support (Strauss \& Corbin, 2006). Results show motivation as the fundamental factor which promotes academic and social integration of the student, which ends in adaptation to the university life. This motivation process is promoted by relationships established by the student with his circle of friends, and it is directly influenced by pedagogic actions developed by teachers, the program, and the university.
\end{abstract}

Keywords: School abandonment, motivation, pedagogy, founded theory. education sciences

*Este artículo presenta resultados de la investigación titulada "Abandono y permanencia estudiantil en el programa de Ingeniería Industrial de la Universidad Francisco de Paula Santander de Cúcuta (UFPS): análisis de factores pedagógicos”, producto de la Tesis de Maestría en Práctica Pedagógica que ofrece la universidad. Este artículo presenta resultados de la investigación titulada "Abandono y permanencia estudiantil en el programa de Ingeniería Industrial de la Universidad Francisco de Paula Santander de Cúcuta (UFPS): análisis de factores pedagógicos”, producto de la Tesis de Maestría en Práctica Pedagógica que ofrece la universidad. 


\section{Introducción}

El fenómeno del abandono académico en la educación superior se ha convertido en un problema relevante para los sistemas educativos en el mundo entero. Este obedece a múltiples causas y representa un elevado costo en el orden económico, social y personal. Las estadísticas sobre abandono arrojan datos abrumadores y son la mejor representación de la magnitud del problema. Según el último informe regional del Instituto Internacional para la Educación Superior en América Latina y el Caribe (IESALC) publicado en 2006, tan solo el $43 \%$ de los estudiantes que inician sus estudios alcanzan la graduación (Ministerio de Educación Nacional [MEN], 2009). Países como República Dominicana, Bolivia y Uruguay alcanzan cifras que superan el $70 \%$; en promedio, los países de América Latina presentan una tasa del $44.5 \%$.

En Colombia la tasa de abandono por cohorte alcanza actualmente un valor cercano al 46\% (Sistema de Prevención y Análisis a la Deserción en las Instituciones de Educación Superior [SPADIES], 2013), considerando los niveles universitario, tecnológico y técnico. Debe aclararse que la tasa de abandono por cohorte implica un seguimiento del historial de los estudiantes retirados por cualquier causa en una cohorte particular, diferenciándose de la tasa de abandono bruta que se calcula por período académico e incluye la totalidad de estudiantes de la institución de educación superior.

Las elevadas cifras en los países latinoamericanos, generan serias reflexiones en cuanto a la efectividad de las estrategias asumidas en política educacional. Por ejemplo, teniendo en cuenta el aumento de cobertura que se ha dado en la educación superior colombiana, los resultados presentan un notorio contraste con los objetivos perseguidos de equidad, democratización y calidad.

Los investigadores Swail, Redd y Perna (2003) realizaron una clasificación de las actividades encaminadas a mejorar la retención estudiantil en cinco categorías: programas de reclutamiento y admisión, de apoyo financiero, de servicios académicos, relacionados con el currículo y la instrucción y programas de servicio estudiantil.
De estas categorías, la que se refiere a los programas relacionados con el currículo y la instrucción sobresale por su importancia en los programas de mejoramiento de la permanencia estudiantil debido a que incorpora la dimensión pedagógica. Los estudios realizados sobre abandono aportan suficiente evidencia acerca de la influencia del componente pedagógico en los procesos de integración del estudiante con la institución educativa, resultados que corroboran la propuesta teórica de Tinto $(1975,1993)$. Es muy importante mencionar que los últimos aportes de Tinto (2006) consideran que la construcción de ambientes significativos de aprendizaje en el aula así como la participación del estudiante en comunidades académicas, aumentan las probabilidades de persistencia y permanencia. Es notable la importancia dada por este investigador a las prácticas pedagógicas al considerar que los factores determinantes para la permanencia se encuentran en el aula.

Moreno, Pedraza y Pineda (2010) desarrollaron un estudio de corte mixto en instituciones de educación superior colombiana con el objetivo de identificar y analizar las acciones pedagógicas que favorecen la permanencia de los estudiantes. Los resultados del estudio permiten identificar que una de ellas es la vinculación y el compromiso del estudiante con su formación académica y social es la participación en comunidades de aprendizaje. La relación docente-estudiante dentro de estas comunidades fomenta el compromiso del estudiante con su formación intelectual y personal, además de hacerlo sentir reconocido y aceptado. Para las instituciones de educación superior, un docente ideal debe poseer el saber disciplinar, capacidades investigativas y estar abierto al diálogo y comunicación con los estudiantes.

Los estudios mencionados revelan la trascendencia que adquiere la dimensión pedagógica en la búsqueda de soluciones al complejo fenómeno del abandono. Vale la pena mencionar que en nuestro país son muy escasas las investigaciones realizadas en las que se estudie el papel que pueden desempeñar las prácticas pedagógicas en la creación de ambientes y contextos de aprendizaje significativos, así como su contribución en aspectos tales como el desarrollo de habilidades cognitivas y de pensamiento superior, desarrollo de competencias emocionales y comunicativas o en el desarrollo moral y espiritual. 
La literatura existente sobre trabajos investigativos referentes al abandono, persistencia y permanencia es abundante en estudios de corte explicativo que identifican factores determinantes de tipo académico, individual, socio-económico e institucional. Los resultados de estos estudios fundamentan la toma de decisiones en políticas de carácter gubernamental e institucional, que generalmente ignoran las múltiples dimensiones implicadas en la problemática del abandono estudiantil.

Por lo tanto, se hace necesario el desarrollo de investigaciones de corte comprensivo que permitan la construcción de conocimiento en torno a este tema en el ámbito latinoamericano, que contribuyan a la formulación de modelos teóricos que afronten esta temática desde perspectivas diferentes, especialmente desde una visión más humana. En este sentido, el desarrollo del presente estudio estuvo motivado por el deseo de colaborar en la generación de ese conocimiento tan necesario para la comprensión del fenómeno del abandono académico en el contexto local de la ciudad de Cúcuta, específicamente en el programa de Ingeniería Industrial de la Universidad Francisco de Paula Santander, institución de carácter oficial que cuenta en la actualidad con aproximadamente 20 mil estudiantes.

El Programa de Ingeniería Industrial de la Universidad Francisco de Paula Santander fue creado en el año 2006, es uno de los programas académicos que cuenta con mayor demanda por parte de los jóvenes norte santandereanos, contando actualmente con una población de 850 estudiantes. En la actualidad, este programa se encuentra desarrollando el proceso de autoevaluación con miras a obtener de parte del Ministerio de Educación Nacional la Acreditación de Alta Calidad. Los datos más recientes (Spadies, 2013) indican que la tasa de abandono por cohorte en la Universidad Francisco de Paula Santander alcanza el $57 \%$, cifra que supera la media nacional que se encuentra en un $46 \%$. Por su parte, en el Programa de Ingeniería Industrial la tasa de abandono por cohorte se estima en un $41 \%$.

En el presente artículo se exponen los resultados del análisis de los factores pedagógicos y su influencia sobre los fenómenos de abandono y permanencia académica en el Programa de Ingeniería Industrial, un estudio de corte comprensivo que utilizó la metodología cualitativa de la Teoría Fundamentada.

\section{Materiales y métodos}

La estructura del diseño de investigación estuvo conformada por las siguientes fases: preparatoria, trabajo de campo, análisis de la información y elaboración del informe final. Estas etapas no presentan una secuencia lineal, por el contrario, su comportamiento es de naturaleza cíclica, donde eventualmente cada una de las fases puede superponerse con las demás y colaborar en su reformulación y reestructuración (Rodríguez, Gil \& García, 1996).

\section{Fase preparatoria}

La investigación se construyó con base en tres líneas de apoyo: ontológica, epistemológica y metodológica (Jaramillo \& Murcia, 2008). Desde la perspectiva ontológica se asumió la ontología sistémica al considerar el objeto de estudio como una estructura o sistema con un alto nivel de complejidad. Epistemológicamente el estudio se fundamentó en el paradigma post-positivista; este establece que el conocimiento es el resultado de un proceso dialéctico entre el sujeto investigador y el objeto de estudio (Martínez, 2006). Las líneas de apoyo teóricas del estudio estuvieron conformadas por la Teoría del Interaccionismo Simbólico, la Teoría Fundamentada y los desarrollos teóricos de Vincent Tinto. Finalmente, la metodología asumida en el estudio fue la indicada por la Teoría Fundamentada, derivada de la Teoría del Interaccionismo Simbólico, que permite la comprensión de los sentidos y significados de las experiencias humanas para generar teoría inductiva sobre un área substantiva (Glaser \& Strauss, 1967).

Se realizó una delimitación de la población considerando los estudiantes de los tres primeros semestres del Programa de Ingeniería Industrial (415 estudiantes). Debe aclararse, que para efectos del presente estudio, se entiende como primer semestre al período lectivo correspondiente al primer semestre del año 2012.

La selección de los participantes fue realizada mediante muestreo intencional y durante el desarrollo 
de este proceso se eligieron trece (13) estudiantes de diferente rendimiento académico, con el fin de disponer de la mayor variabilidad posible en la información tal y como lo establece la metodología de la Teoría Fundamentada.

Técnica de recolección de la información: Se eligió la entrevista en profundidad teniendo en cuenta que esencialmente permite la interacción con los sujetos de la investigación, facilitando el acceso a la realidad de las experiencias humanas con el fin de comprender sus sentidos y significados (Carrero, Soriano \& Trinidad, 2006). Las entrevistas fueron realizadas en forma grupal.

\section{Trabajo de campo}

Con el fin de considerar la máxima variabilidad en la muestra, se clasificaron inicialmente los informantes potenciales con base en su promedio de notas, en tres categorías de rendimiento académico: alto, mediano y bajo. Se realizaron un total de cinco (5) entrevistas grupales para un total de trece (13) estudiantes. El tamaño de estos grupos fue de dos, tres y cuatro estudiantes. Los grupos fueron seleccionados por el investigador de acuerdo con la afinidad observada entre los estudiantes, conocimiento adquirido como consecuencia de haber sido su profesor y de tal forma que se cubriera todo el rango de variabilidad establecido para el rendimiento académico. Las entrevistas fueron realizadas en la biblioteca Eduardo Cote Lamus de la Universidad Francisco de Paula Santander y fueron grabadas con autorización de los participantes.

Los procesos de recolección y análisis de la información se desarrollaron en forma simultánea, atendiendo a la lógica de la investigación que utiliza métodos cualitativos. Por tal motivo, una vez finalizadas las entrevistas, se procedía al proceso de transcripción de la misma con el fin de realizar los análisis establecidos por la Teoría Fundamentada. El proceso mismo de muestreo estuvo retroalimentado por los resultados de estos análisis, los cuales generaban códigos substantivos que indicaban al investigador la necesidad de elegir los participantes apropiados con el fin de recolectar la información necesaria para continuar con la aplicación del Método Comparativo Constante y de este modo alcanzar la saturación teórica.

\section{Análisis de la información}

Las entrevistas se transcribieron y se analizaron utilizando el software Atlas.ti versión 5.2. Cada entrevista suministraba datos que eran clasificados mediante codificación abierta, comparando cada incidente y estableciendo categorías. El procedimiento indicado por el Método Comparativo Constante permitió la búsqueda de semejanzas y diferencias en la información para obtener propiedades y dimensiones de las diferentes categorías que se iban generando. Estos resultados orientaban la búsqueda de información mediante la selección de nuevos participantes con el fin de alcanzar la saturación teórica. (Carrero, Soriano \& Trinidad, 2006). Es importante mencionar que durante todo el proceso de análisis de la información se iban introduciendo memos teóricos y analíticos que estaban relacionados con situaciones de relevancia que se iban descubriendo durante el desarrollo y análisis de las entrevistas.

Este proceso de codificación permitió obtener inicialmente un listado de familias de códigos substantivos. Posteriormente, a medida que se continuaba con el desarrollo de la investigación y con la aplicación del Método Comparativo Constante, se logró un mayor conocimiento de las propiedades de las categorías y sus regularidades lo que condujo a un nivel de abstracción más elevado que permitió el surgimiento de códigos conceptuales mediante la combinación, reducción e integración de categorías.

Esta transformación de códigos substantivos en conceptuales se convirtió en la etapa fundamental del análisis, porque se trataba de encontrar un sentido a las diversas relaciones que habían surgido entre las categorías y a la creación de una estructura que soportara todo el análisis realizado hasta ese momento. Este periodo de gran relevancia en la investigación condujo al descubrimiento de la categoría central que en este estudio también se indica mediante un código "in vivo" (Glaser, 1978). A partir del descubrimiento de la categoría central se reconocieron dos procesos que son inseparables, están interconectados y facilitan la comprensión de las acciones e interacciones que se presentan en el contexto de la vida universitaria. Finalmente, la categoría central y los procesos permiten la integración de los códigos conceptuales en un modelo o estructura teórica que da un sentido y significado a la realidad del fenómeno estudiado. 


\section{Resultados}

La combinación, reducción e integración de las categorías permitió la emergencia de los códigos conceptuales y el descubrimiento de la categoría central con su código "in vivo" "Mi proyecto de vida". Dos procesos fueron identificados: la motivación y adaptación, los cuales ayudan en la comprensión de la complejidad del fenómeno analizado.

El análisis basado en el desarrollo conceptual permitió la construcción de un modelo o estructura teórica. Todo inicia con la transición Colegio Universidad que implica la elección de la carrera Ingeniería Industrial. En esencia, la transición es un proceso de incertidumbre donde intervienen diversos factores, especialmente subjetivos. Después viene la nueva vida en la universidad donde tiene lugar la interacción con la realidad académica, social y estructural de la institución. Es el espacio y el tiempo donde se desarrollan los procesos de motivación y adaptación del estudiante, métodos que son soportados y apoyados por las acciones pedagógicas de los profesores, del programa y de la universidad, pero muy especialmente por las relaciones que el estudiante establece con su círculo de amigos más cercano, que se convierte en un factor potenciador de la motivación por el estudio y la superación personal.

El proceso de motivación desempeñó un papel trascendental en el desarrollo de la investigación y se convirtió en el factor clave para la comprensión de la realidad estudiada. La motivación es el factor fundamental que impulsa el trabajo y el desarrollo académico y personal del estudiante que culmina con la adaptación a la vida universitaria. En este sentido, se trata de un individuo que es consciente de su proceso de construcción como ser autónomo y estructurado, que lucha por alcanzar sus metas fijadas, "su proyecto de vida" y comienza a visionar la prospectiva de sus acciones. Esta visión comprende la percepción del profesor, del Programa de Ingeniería Industrial, de la Universidad Francisco de Paula Santander y de sí mismo. Por último, toda esta realidad está inmersa dentro de un proceso educativo cuya finalidad es la formación y el desarrollo del ser humano; es aquí donde se reconoce la realidad del otro y se hace presente la acción moral del educador, contribuyendo en el desarrollo de un proyecto humanista que se basa en la comprensión y en la solidaridad (Gadamer, 2000).

\section{Discusión de resultados}

El escenario de la universidad presenta una realidad cultural, social y académica que plantea al estudiante el reto de generar estrategias que permitan su adaptación. Del éxito en lograr esta adaptación depende el progreso personal y el aproximarse a la meta deseada. En cuanto a la realidad social, el estudiante forma parte de una población heterogénea de jóvenes con diversas formas de pensar, sentir y actuar. Durante el primer semestre, el estudiante da el primer paso en el proceso de adaptación social que consiste en lograr la formación de un círculo de amigos, con quienes comparte metas, gustos, estilo de vida, modelos mentales, tiempo de clases y tiempo de estudio. Es notable la influencia que tiene el grupo o círculo de amigos en el proceso de adaptación o vinculación a la vida universitaria.

Esta influencia cobra más relevancia al considerar el reto principal al que se enfrenta el estudiante, que consiste en responder a las exigencias académicas propias del programa y de la universidad. Existe la necesidad no solo de lograr nuevos aprendizajes sino de superar la evaluación de los mismos. Se genera una dinámica en el joven que lo conduce a la construcción de su autonomía mediante el esfuerzo, la disciplina de estudio y la motivación propia con el fin de alcanzar el éxito académico. Además, es aquí donde se visualiza la estrategia más favorable para la adaptación académica de los jóvenes que consiste en buscar apoyo y soporte en el círculo cercano de amigos. La modalidad de estudio en grupo les permite unirse para compartir conocimientos, ser generosos y solidarios, animarse a estudiar y superar las dificultades. De esta manera, aparece el trabajo colaborativo como soporte del aprendizaje y la superación académica.

¿Qué es lo que mueve a los estudiantes a unirse para sobrevivir a las exigencias propias de la academia, lograr la superación y continuar con el desarrollo de su proyecto de vida? ¿Cómo se genera esa fuerza que impulsa a estos jóvenes a seguir adelante? Es importante comprender que el proceso fundamental que subyace a la estrategia del aprendizaje colaborativo es la motivación, que se convierte 
en el factor que impulsa el desarrollo académico y personal del estudiante y una clave para entender todo el proceso de adaptación al mundo universitario.

Cuando se analizan las razones que movilizan a los jóvenes hacia el aprendizaje colaborativo, hacia la solidaridad, la generosidad, el compromiso y la satisfacción de servir a los demás, se llega a los descubrimientos recientes de la neurociencia que indican que la razón no existe en forma separada de los sentimientos y emociones, sino que constituyen un sistema cognitivo-afectivo (Damasio, 1994). Avanzando un poco más, se llega al concepto de motivación intrínseca, que se define como: "El deseo de ocuparse en una actividad por su propia esencia, es decir, tan solo por la satisfacción que da" (Kohn, 1993:87). A diferencia de la motivación extrínseca en donde el incentivo que orienta la conducta es independiente de la actividad realizada, en la motivación intrínseca, este es inherente a la actividad misma y no necesita reforzamiento. Además, se ha determinado que el apoyo emocional y la capacidad para establecer relaciones personales operan como condicionantes de la motivación intrínseca (Deci \& Ryan, 2000).

Damasio (1994) ha planteado la hipótesis de los marcadores somáticos con el fin de explicar la aproximación que realiza el ser humano a su dominio social más inmediato. Los marcadores somáticos son sentimientos que se generan mediante las emociones y están relacionados con la toma de decisiones al enfrentarse a la vida social. Existe una profunda relación entre mente, emociones, afecto y visión a largo plazo.

A pesar de la diversidad de perspectivas teóricas que presenta un constructo como la motivación, el tratar de comprender y descubrir las razones que nos impulsan a la acción, a continuar luchando por nuestra superación, en el caso de los estudiantes a lograr la adaptación y alcanzar el título de profesional está estrechamente vinculado con esa visión a largo plazo y la percepción de que solo mediante la constancia y el empeño en mantener esa actitud se podrá alcanzar el éxito. Se trata de una situación bastante compleja en donde se presenta la interacción del individuo con la sociedad y la motivación logra liberar las capacidades latentes del ser humano que lo conducen al cambio y a la transformación personal.
Los procesos de motivación y superación del estudiante reciben la influencia directa de las acciones pedagógicas desarrolladas por los profesores, el programa y la universidad. De tal forma, que la naturaleza de estas acciones resulta determinante para lograr la comprensión e identificación de las complejas relaciones que se presentan durante el proceso de adaptación a la vida universitaria.

Las prácticas pedagógicas de los profesores ejercen un efecto considerable en la motivación de los estudiantes de Ingeniería Industrial. Factores pedagógicos como los contenidos significativos de aprendizaje, la metodología adoptada en las clases, la claridad en la exposición, las relaciones que se establecen con el estudiante, el fomento del aprendizaje colaborativo y la metacognición, la naturaleza de las tareas y trabajos asignados, la orientación y acompañamiento del trabajo del estudiante, entre otros; ejercen una profunda influencia en el proceso de adaptación académica del estudiante a la vida universitaria, principalmente debido a que tienen un efecto considerable en la motivación y vinculación.

Los contenidos de aprendizaje y las metodologías implementadas por los docentes en sus clases están asociados con experiencias significativas que son muy importantes para los estudiantes, porque permiten encontrar un sentido y significado al estudio realizado, lo que conduce a la motivación y al interés por seguir avanzando en el camino del aprendizaje y de la formación. Las metodologías utilizadas por los docentes pueden ser muy valiosas para la creación de ambientes y experiencias significativas en la medida en que se fomente la participación, la interacción, el aprendizaje colaborativo y el desarrollo de procesos de pensamiento superior.

La claridad en la exposición está directamente relacionada con el uso de estrategias pedagógicas y didácticas que faciliten la comprensión de las diferentes temáticas que se estudian; es un factor relevante porque implica la construcción del conocimiento, el desarrollo de estructuras cognoscitivas del estudiante.

Las relaciones que establecen profesores y estudiantes están asociadas con el desarrollo intelectual y humano. La dinámica de estas relaciones adquiere una gran relevancia debido a que los procesos formativos se 
fundamentan en la comprensión y el reconocimiento del otro, la posibilidad de dejarse impresionar por la realidad ajena. Debido a que la comprensión del otro se hace posible a través de la intermediación del lenguaje (Gadamer, 2000), es mediante el diálogo pedagógico entre el profesor y el estudiante que se construye un proyecto de humanidad, porque se supera la propia individualidad para escucharse, para eliminar las distancias y trabajar solidariamente con el fin de alcanzar metas comunes. Esta experiencia profundamente humana es trascendente y alcanza los niveles de la experiencia estética (Gadamer, 2000), de tal forma que la práctica pedagógica como elección moral del profesor reivindica la naturaleza esencial de la educación como forjadora de horizontes de posibilidad y de sentido para los seres humanos.

Las acciones pedagógicas desarrolladas por el programa y la universidad también influyen en el proceso de motivación de los estudiantes. Estas están enfocadas hacia lo académico, cultural y deportivo con la finalidad de promover la adaptación a la vida universitaria. Los procesos comunicativos que se establecen entre el Programa de Ingeniería Industrial, la universidad y los estudiantes tienen una gran importancia debido a la necesidad de disponer de una información adecuada respecto a la realidad de la institución y sus actividades, lo que conduce a la apropiación y al sentido de pertenencia por la institución y el programa. Existe otro frente de acción igualmente relevante que está relacionado con el apoyo y soporte profesional dado por el programa y la universidad a los estudiantes y la gestión desarrollada para lograr la vinculación con el entorno social y laboral. Este conjunto de actividades está asociado con actividades de formación y desarrollo profesional que resultan indispensables para enfrentarse a la realidad de la vida laboral.

El trabajo del estudiante con miras a lograr la superación personal, educarse, construirse como ser humano estructurado, reflexivo y autónomo, forma parte de un proyecto de vida que indica el sendero a recorrer para alcanzar el éxito como persona y como profesional. En este sentido, el estudiante posee una prospectiva de sus acciones que está determinada por la percepción que tiene de los profesores, del programa, de la universidad y de sí mismo como ser en permanente formación. Este hallazgo de la investigación resulta interesante porque conduce a una visión conjunta, a un proyecto común de construcción de humanidad que está fundamentado en el plan formativo de la universidad.

Los estudiantes perciben al profesor ideal como un pedagogo, un guía, la persona que orienta e ilumina el camino que conduce al conocimiento. Este es ante todo un humanista que reconoce y percibe al estudiante como un ser que desea formarse y avanzar en su proceso de construcción personal.

¿Cómo deberían ser la universidad y el Programa de Ingeniería Industrial de acuerdo con esta visión prospectiva de los estudiantes? ¿Cuáles son los procesos que deberían desarrollarse para lograr las metas anheladas por estos jóvenes? Ante todo, la universidad y el programa deben ser responsables del desarrollo intelectual y humano, procurando priorizar la academia, la creación de un nivel cultural y un ambiente de convivencia apropiados que permitan la integración y adaptación de sus estudiantes. Es una concepción de la universidad como institución comprometida con el desarrollo de acciones pedagógicas, para modificar esquemas y conductas que son el reflejo de una ciudad que tiene un nivel cultural muy bajo y un ambiente de convivencia bastante crítico, consecuencia de múltiples causas como son el efecto nocivo de los medios de comunicación, la ausencia de liderazgo político, social, gremial y económico, el desprecio e irrespeto por las leyes y normas de convivencia, entre otras. Para la universidad y el programa representa el reto de formar ciudadanos integrales con un potencial académico y humano que permita llevar a cabo las transformaciones que la sociedad necesita y que aparecen explícitas en la misión de la institución.

Por último, ¿cuál es la visión del sí mismo? ¿Cuál es la prospectiva de la labor que se desarrolla como estudiante universitario? El reconocimiento del sí mismo como condición indispensable del proceso formativo (Campo \& Restrepo, 2000), es un indicador de la construcción del proyecto de vida del estudiante, de la necesidad de recorrer el sendero que conduce a las metas anheladas, de la búsqueda de la autonomía y de la superación intelectual, moral y espiritual del individuo que encuentra un sentido a su existencia a través del acto educativo. Se refiere al ser como 
posibilidad, como una construcción permanente, un proyecto de vida que se convierte en un proyecto común de humanidad cuando se consideran los otros, sus compañeros, quienes también transitan por ese sendero que traza la educación.

\section{Conclusiones}

El análisis interpretativo que fundamenta la metodología de la Teoría Fundamentada, permitió la comprensión de la influencia de los factores pedagógicos en los fenómenos del abandono y permanencia de los estudiantes de Ingeniería Industrial de la Universidad Francisco de Paula Santander. Esta comprensión se obtuvo debido a que la investigación estuvo orientada a develar los significados y sentidos que tienen para los estudiantes las prácticas pedagógicas de los profesores así como las acciones formativas desarrolladas por el programa y la universidad.

El proceso de motivación desempeñó un papel trascendental en el desarrollo de la investigación y se convirtió en el factor clave para la comprensión de la realidad estudiada. Este es el factor fundamental que impulsa el trabajo y el desarrollo académico y personal del estudiante que culmina con la adaptación a la vida universitaria. En este sentido, la totalidad de los factores pedagógicos que intervienen en las acciones desarrolladas tanto por los profesores como por el programa y la institución, deben estar orientados a la liberación de las capacidades latentes y del potencial humano de los estudiantes a través del fomento de la motivación. Facilitar este proceso es una responsabilidad humana y ética que conduce a la transformación del individuo que encuentra un significado y sentido a su existencia a través del proceso educativo, desarrollando de esta forma su proyecto de vida.

Se identificaron las siguientes dificultades a las que se enfrentan los estudiantes en su proceso de adaptación a la institución universitaria: el desconocimiento y temor a la vida universitaria, los escasos conocimientos previos en las asignaturas de Ciencias Básicas y antecedentes de bajo rendimiento académico, el desafío planteado por la exigencia académica propia del programa y finalmente las deficiencias observadas no solo en las prácticas pedagógicas de los profesores, sino también en las acciones y procesos desarrollados por el programa y la universidad.

Al ingresar a la universidad el estudiante se enfrenta a una realidad social, académica y cultural que es desconocida y ante la cual debe generar estrategias con el fin de lograr la adaptación. Los análisis realizados permitieron reconocer las siguientes estrategias: el esfuerzo y superación personal, la formación de un círculo de amigos y el soporte dado por las acciones pedagógicas de los profesores, el programa y la universidad.

Durante el primer semestre el estudiante inicia el proceso de adaptación social mediante la formación del círculo de amigos. Pero este no solo facilita la adaptación social, también contribuye significativamente a la adaptación académica debido a que sirve de apoyo y soporte para responder a las exigencias académicas del programa. De esta manera, aparece el trabajo colaborativo como soporte del aprendizaje y la superación académica. Es una estrategia basada en la solidaridad que dinamiza los procesos de estudio e integración fomentando la motivación, el proceso clave en este estudio, el cual está estrechamente relacionado con el proceso de adaptación del estudiante.

La realidad estructural y académica de la universidad es un factor fundamental para la adaptación y permanencia, debido a la influencia que tienen las prácticas pedagógicas de los profesores y las acciones formativas del programa y de la universidad en la motivación del estudiante. De la información recolectada a través de las entrevistas, puede afirmarse que las prácticas de los profesores desempeñan un papel de relevancia en lo que se refiere a la creación de ambientes significativos de aprendizaje, fomento de la comprensión, desarrollo de procesos cognitivos, acciones de acompañamiento y asesoría, contribución al desarrollo intelectual y humano, así como en la motivación y vinculación del estudiante con la realidad universitaria. Es decir, las relaciones pedagógicas que se establecen entre los profesores y los estudiantes, se convierten en el factor fundamental del proceso de formación académica y humana que conduce a la adaptación de los estudiantes. 
Es conveniente que los docentes desarrollen contenidos e implementen metodologías en sus clases tendientes a fomentar la participación, la interacción, el aprendizaje colaborativo, el desarrollo de procesos de pensamiento superior y la generación de ambientes significativos de aprendizaje que son muy importantes para los estudiantes, porque les permite encontrar un sentido y significado al estudio realizado, lo que conduce a la motivación y al interés por seguir avanzando en el camino del aprendizaje y de la formación. Los estudiantes expresan la necesidad de relacionar los contenidos que se enseñan en clase con la aplicabilidad de los conocimientos.

La claridad en la exposición está directamente relacionada con el uso de estrategias didácticas que faciliten la comprensión de las diferentes temáticas que se estudian; es un factor relevante porque implica la construcción del conocimiento, el desarrollo de estructuras cognoscitivas del estudiante. Las deficientes acciones pedagógicas y didácticas conducen a la desmoralización y desmotivación por el estudio de las asignaturas. Por tal motivo, se recomienda el diseño y planificación de estrategias didácticas orientadas a la formación del pensamiento crítico disciplinar que implica el desarrollo de las cuatro dimensiones: lenguaje y argumentación, solución de problemas, metacognición y motivación.

Las relaciones que establecen profesores y estudiantes se convierten en un factor de gran trascendencia debido a que los procesos formativos se fundamentan en la comprensión y el reconocimiento del otro, en la posibilidad de dejarse impresionar por la realidad ajena. En este sentido, se hace necesario que los docentes implementen en sus prácticas pedagógicas estrategias encaminadas a la generación de ambientes que favorezcan el diálogo, la confianza y la interacción personal. De esta forma el acto pedagógico que implica la relación dialéctica maestro-alumno permite la apropiación del saber y la transformación del individuo.

Los estudiantes perciben al profesor ideal como un pedagogo, como un guía, la persona que orienta e ilumina el camino que conduce al conocimiento. Este pedagogo es ante todo un humanista que reconoce y percibe al estudiante como un ser que desea formarse, que desea avanzar en su proceso de construcción personal.
Las tareas y actividades asignadas a los estudiantes deben estar diseñadas de tal forma que contribuyan al desarrollo de capacidades intelectuales y procesos de pensamiento superior, las cuales adquieren una gran importancia en el proceso de formación integral de un profesional que se debe desempeñar en el mundo globalizado y post-tecnológico del siglo XXI. Esas actividades expresan la capacidad que tiene el profesor para generar interés y motivación por el aprendizaje.

La imagen deseada del profesor como persona que acompaña y orienta, es decir, como el pedagogo que está dispuesto a observar las anomalías en el desarrollo del proceso educativo, a detenerse y reflexionar con la finalidad de realizar una lectura adecuada de la realidad, se puede apreciar en las declaraciones de los estudiantes cuando expresan la necesidad de recibir una orientación adecuada en el proceso de aprendizaje por parte del docente, de ser encaminados por la ruta correcta hacia el conocimiento.

Las acciones pedagógicas desarrolladas tanto por el Programa de Ingeniería Industrial como por la universidad, también ejercen una influencia considerable en la generación de motivación e interés en los estudiantes. Las actividades académicas, culturales y deportivas, los procesos comunicativos, las relaciones que establecen el programa y la universidad con el entorno social y laboral, junto con el apoyo y soporte profesional dado a los estudiantes, se constituyen en actividades significativas de motivación que inciden directamente en la permanencia y en el proceso formativo.

La universidad y el programa deben ser responsables del desarrollo intelectual y humano, procurando priorizar la academia, la creación de un nivel cultural y un ambiente de convivencia apropiados que permitan la integración y adaptación de sus estudiantes. Es una concepción de la universidad como institución comprometida con el desarrollo de acciones pedagógicas para modificar esquemas y conductas que son el reflejo de una ciudad que tiene un nivel cultural muy bajo debido a múltiples causas. Para la universidad y el programa representa el reto de formar ciudadanos integrales con un potencial académico y humano que permita llevar a cabo las transformaciones que la sociedad necesita y que aparecen explícitas en la misión de la institución. 
Es imperativo que la universidad y el programa establezcan unos procesos comunicativos más eficientes, de tal manera que la información necesaria esté disponible favoreciendo los procesos de integración académica y social. Es una situación preocupante debido a que los estudiantes manifiestan que desconocen los lineamientos del programa y de la universidad, las políticas de investigación, los sistemas de información y los avances logrados en el proceso de acreditación entre otros. Situación que debe tenerse muy presente en el desarrollo de los procesos de autoevaluación con fines de acreditación que adelanta la institución en la actualidad.

Las acciones que se consideran más necesarias para la formación y el desarrollo profesional son aquellas que involucran la gestión del programa y de la universidad, para lograr la vinculación con el contexto social e industrial de la región. Es una concepción generalizada en los estudiantes, quienes reclaman el compromiso institucional para que la universidad les preste apoyo y soporte en diferentes actividades como las prácticas profesionales y las visitas industriales. De parte del programa, los estudiantes consideran que se debería promover en los profesores metodologías de enseñanza que hicieran un mayor énfasis en la aplicabilidad de las asignaturas enseñadas debido a que existe un gran interés en el conocimiento de la realidad laboral a la que se debe enfrentar un ingeniero industrial.

\section{Referencias bibliográficas}

Campo, R \& Restrepo, M. (2000). Formación Integral, modalidad de educación posibilitadora de lo humano. Bogotá D.C.: Facultad de Educación, Pontificia Universidad Javeriana.

Carrero, V; Soriano, R \& Trinidad, A. (2006). La construcción de la teoría a través del análisis interpretacional. Madrid: Centro de Investigaciones Sociológicas.

Damasio, A. (1994). Descartes Error. Emotion, Reason, and the Human Brain. New York: Grosset/Putnam.

Deci, E.L. \& Ryan, R.M. (2000a). Intrinsic and extrinsic motivations: classic definitions and new directions. Contemporary Educational Psychology, 25, 54-69.

Deci, E \& Ryan, R. (2000b). Self-determinations theory and the facilitation of intrinsic motivation, social development and well-being. American Psychologist, 55(1), 68-78.

Gadamer, H. (2000). Educar es educarse. Barcelona: Paidós.

Glaser, B \& Strauss A. (1967). The discovery of grounded theory: strategies for qualitative research. New York: Aldine publishing.

Glaser, B. (1978). Theoretical Sensitivity: Advances in the Methodology of Grounded Theory. Mill Valley, C.A: Sociology Press.

Jaramillo, L. \& Murcia N. (2008). Investigación Cualitativa "La Complementariedad". Armenia: Editorial Kinesis.

Kohn, A. (1993). Punished by rewards. The trouble with gold stars, incentive plans, A.s, Praise, and other Bribes. Boston: Houghton Mifflin Company.

Martínez, M. (2006). La Investigación Cualitativa (Síntesis Conceptual). Revista de Investigación en Psicología. Vol. 9, No. 1, 123-146.

Ministerio de Educación Nacional (MEN). (2009). Deserción Estudiantil en la Educación Superior Colombiana. Elementos para su diagnóstico y tratamiento. Bogotá.

Moreno, I; Pedraza, A. \& Pineda, C. (2011) Efectividad de las estrategias de retención universitaria: la función del docente. Educación, Vol. 14, No.1, 119-135.

Rodríguez, G; Gil, J \& García E. (1996). Metodología de la Investigación Cualitativa. Granada: Ediciones Aljibe.

Sistema de Prevención y Análisis a la Deserción en las Instituciones de Educación Superior (Colombia). [En línea]: Sistema de información del Ministerio de Educación Nacional. Disponible desde: http://spadies.mineducacion. 
gov.co/spadies/consultas predefinidas.html?2

Swail, W; Redd, K \& Perna, L. (2003). Retaining minority students in higher education: A framework for success. ASHE-ERIC Higher Education Report, 2 (30), Jossey-Bass Higher and Adult Education Series

Tinto, V. (1975). Dropout From Higher Education: A Theoretical Synthesis of Recent Research. Review of Educational Research , 45, 89-125.

Tinto, V. (1993). Leaving College: Rethinking the Causes and Cures of Student Attrition (2 ed.). Chicago: The University of Chicago Press.

Tinto, V. (2006-2007). Research and practice of student retention: What is next? Journal of College Student Retention, 8, 1-19. 